\title{
Spatial cross-correlation modeling for propagation channels in indoor distributed antenna systems
}

\author{
Li Tian, Xuefeng Yin*, Xu Zhou and Quan Zuo
}

\begin{abstract}
In this contribution, the spatial cross-correlation of composite channels in a distributed antenna system (DAS) is studied for the case that a nine-antenna DAS is deployed in an indoor environment. As few measurement data of DAS propagation channels are available, the propagation graph modeling based on the electromagnetic wave reverberation theory is used to generate the synthetic impulse responses (IRs) for the composite channels induced by the different groups of antennas in the DAS. The simulations take into account the geographic parameters of the environment, the cluttering scatterers, the realistic visibility conditions, as well as the flexible locations for the antennas, and the user equipment. The uncorrelated scattering assumption is proved to be valid by using the real measurement data from the indoor environments. Based on this assumption, narrowband channel cross-correlation is computed by integrating the small-scale fading cross-correlation within the same delay bins in two channel IRs. The characteristics of the cross-correlation in four cases with different antenna grouping are investigated for the nine-antenna DAS. Part of the modeling results are shown to be consistent with the empirical counterparts for specific DAS constellations calculated using the real measurement data.
\end{abstract}

\section{Introduction}

A distributed antenna system (DAS) is referred to as the network of spatially separated antenna nodes connected to a common access point. The DAS has several advantages, comparing with the traditional concentrated multiple-input-multiple-output (MIMO) system (CMS). First, it allows the same coverage of an area with less power consumption since the distributed antennas create line-of-sight (LoS) connections to the mobile station, which can significantly reduce the power consumption caused by wall penetration inside buildings. Also, it reduces the delay spreads of the received signals, as well as the complexity of receivers [1,2]. The locations of antennas can be designed following different principles, e.g., achieving better coverage, enhancing spectral efficiency [3], or increasing the quality of received signals [4].

Recently, the transmission technologies for DAS have attracted a lot of attention [5,6]. For example, in [5], block diagonalization and zero-forcing dirty-paper coding

\footnotetext{
${ }^{*}$ Correspondence: yinxuefeng@tongji.edu.cn

College of Electronics and Information Engineering, Tongji University,

Shanghai, China
}

\section{Springer}

downlink transmission schemes are extended for a singlecell DAS with multi-antenna-distributed antenna nodes. Only a subset of the antenna nodes in the cell transmits to the user equipment (UE). The aggregate cell spectral efficiency achieved by the proposed schemes per frequencytime resource block is compared for both DAS and CMS architectures. It has been demonstrated that, under the same total power constraint, the cellular DAS can double the ergodic aggregate cell spectral efficiency of the CMS. In addition, the spectral efficiency increases and saturates as the number of antennas per node increases. In [6], a generalized DAS combined with the CMS is introduced, where each distributed node has multiple micro-diversity antennas. All the antennas have a separate feeder to the base station. The signals can be combined using different algorithms.

For the existing theoretical studies of DAS, oversimplified propagation channel models and ideal assumptions are usually adopted for the performance analysis [6]. The impact of real environment on the DAS performance has not been thoroughly investigated. In these simplified channel models, the power of a single channel at each 
antenna in a distributed antenna node follows the chisquared distribution with two degrees of freedom $\left(\chi_{2}^{2}\right)$, while the mean of the distribution varies independently with log-normal distribution. The average of stochastic power means of the antennas within a node is determined by the path loss from the UE to the node, neglecting the shadow fading attributed to the UE surroundings. The 'inter-node' and the 'intra-node' fadings are assumed to be independent and identical log-normal and Rayleigh distributions, respectively. According to [6], the independence of the 'inter-node' fading is justifiable by the large distance between any two nodes and the distinctive scattering around the antennas in each node. However, as illustrated in [7], propagation environments, particularly the indoor propagation environment, introduce significant dependence among the nodes even when these aforementioned conditions are satisfied. Therefore, field measurements or realistic simulations are essential for establishing DAS channel models in both macroscopic and microscopic view points.

In this contribution, a simulation approach is applied to analyzing the characteristics of fading correlation across multiple links in DAS scenarios. The electromagnetic (EM) wave reverberation theory, originally adopted in [8] for generating channel impulse response (IR), is adopted to create the synthetic IRs in DAS scenarios. The spatial cross-correlations are then investigated by taking into account specific DAS configurations in an indoor environment. The validity of the predicted characteristics is evaluated using measurement data.

The organization of this paper is as follows: section 1 gives the general background of the works; in section 2, the approach of DAS channel simulations based on propagation graph are elaborated, and the simulations are validated using measurement data; in section 3 , the spatial cross-correlation for DAS channels is defined; the simulation results for an indoor scenario are presented in section 4; experimental confirmation of the characteristics predicted by the simulations is depicted in section 5; finally, conclusive remarks are addressed in section 6 .

\section{Propagation graph modeling for DAS channels}

\subsection{A brief introduction of graph modeling}

The propagation graph modeling approach has been applied to predicting channel IRs in [8] by exhaustively searching of the propagation paths connecting the transmitters and receivers. A propagation graph can be generated by taking into account the geometry of the environment, the scatterers' distribution, the mobility, and visibility of the scatterers, as well as the scatterers' EM properties.

For example, as shown in Figure 1, if the position and visibility information of transceivers and scatterers has

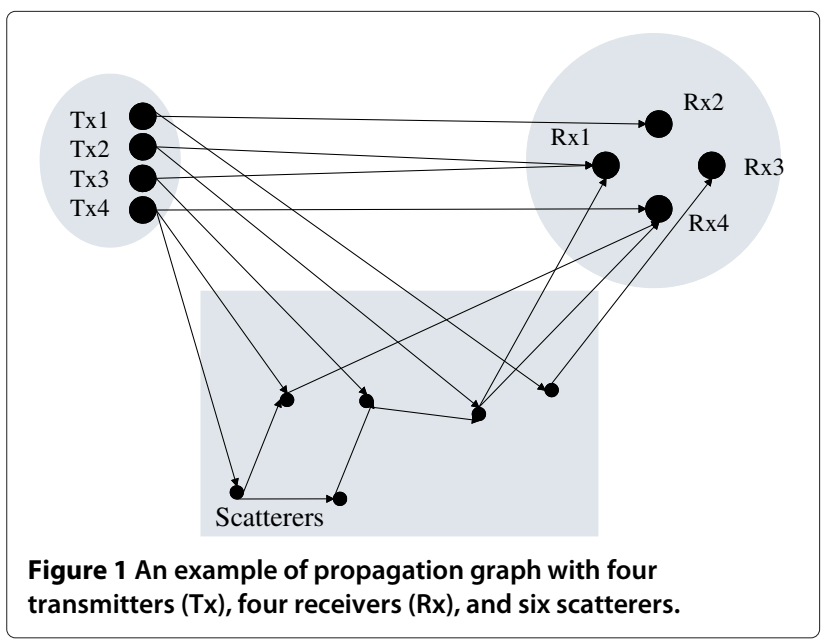

been configured, the transfer function of the propagation can be calculated in the frequency domain as [8]:

$$
\begin{aligned}
\mathbf{H}(f)= & \mathbf{D}(f)+\mathbf{R}(f)\left[1+\mathbf{B}(f)+\mathbf{B}^{2}(f)\right. \\
& \left.+\cdots+\mathbf{B}^{n}(f)+\cdots\right] \mathbf{T}(f) \\
= & \mathbf{D}(f)+\mathbf{R}(f)[1-\mathbf{B}(f)]^{-1} \mathbf{T}(f),
\end{aligned}
$$

where $\mathbf{D}(f)$ represents the LoS part of the transmission, and $\mathbf{R}(f)[1-\mathbf{B}(f)]^{-1} \mathbf{T}(f)$ is the none line-ofsight (NLoS) component induced by the reverberation of EM waves among scatterers. In Equation 1, $\mathbf{T}(f)$, $\mathbf{R}(f)$, and $\mathbf{B}(f)$ denote the transmission matrices with entries representing the power attenuations and phase changes from individual transmitter ( $\mathrm{Tx}$ ) to scatterers, from scatterers to individual receiver $(\mathrm{Rx})$, and among scatterers, respectively. $\mathbf{B}^{n}(f)$ refers to the $n$th bounce inter-reflections of the scatterers. The transfer function of a propagation path that represents a link in Figure 1 can be calculated as:

$$
A_{\mathrm{e}}(f)=g_{\mathrm{e}}(f) \exp \left(j 2 \pi \tau_{\mathrm{e}} f+j \phi\right),
$$

where $A_{\mathrm{e}}$ is an element in the matrics $\mathbf{D}, \mathbf{T}, \mathbf{B}$, and $\mathbf{R}$ according to different kinds of link ends, $g_{\mathrm{e}}(f)$ is the propagation coefficient calculated based on the free-space propagation loss and the reflection coefficient, $\tau_{\mathrm{e}}$ is the delay or time of arrival, and $\phi$ is the random phase rotation, which follows a uniform distribution on the interval $[0,2 \pi)$.

The graph modeling approach is adopted in this research because of the following advantages: firstly, an environment can be easily modeled by the location matrices which contain the location information of the transceivers and scatterers; secondly, IRs are calculated with analytical computations based on the EM-wave reverberation theory; thirdly, the models can be generalized for specific frequency bands. Moreover, the complexity of graph modeling approach is limited. For example, IR generation for a graph with 2 transmitters, 2 receivers, and 


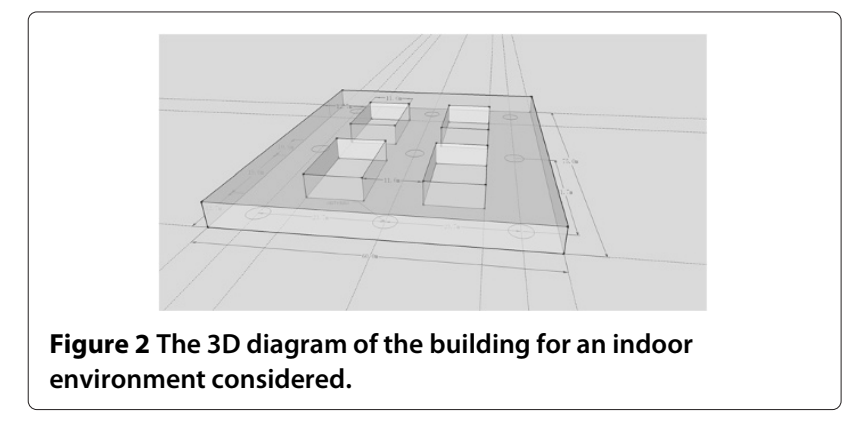

100 scatterers in a snapshot costs about $8 \times 10^{6}$ flops which can be executed in less than $0.1 \mathrm{~s}$ using the computing software, e.g., MATLAB ${ }^{\prime \prime}$ with a quad-core computer. In addition, the IR obtained by the graph approach exhibits the specular-to-diffuse transition, which is hard to obtain using conventional ray-tracing-based channel modeling with tractable complexity [9-11].

The effectiveness of applying the graph approach to channel modeling has been investigated in [8] and [12], where the power-delay profiles and the specular-todiffuse transition of the simulated IRs are compared with those of the real IRs. In [13], the spatial and temporal characteristics of the channel IRs generated by graph in high-speed railway scenarios have been shown to be realistic. In this contribution, the graph modeling approach is further evaluated in an indoor scenario by comparing the statistical characteristics of channel parameters obtained from graph modeling with the parameter values specified in established WINNER II channel models [14]. It will be shown later in section 2.2 that the typical channel characteristics predicted by the graph modeling is consistent with the WINNER II models.

\subsection{Evaluation of propagation graph modeling}

An indoor hall environment as depicted in Figure 2 is considered for evaluating the applicability of graph-modeling in reproducing realistic channel characteristics. The hall is 60-m wide, 70-m long, and 5-m high. Within the hall, a number of rectangular structures exist, which attenuate the EM waves dramatically and have significant impact on the visibility among the scatterers and the antennas. In total, nine distributed antennas are deployed in the hall. The locations of the antennas are marked with ' $\nabla$ '

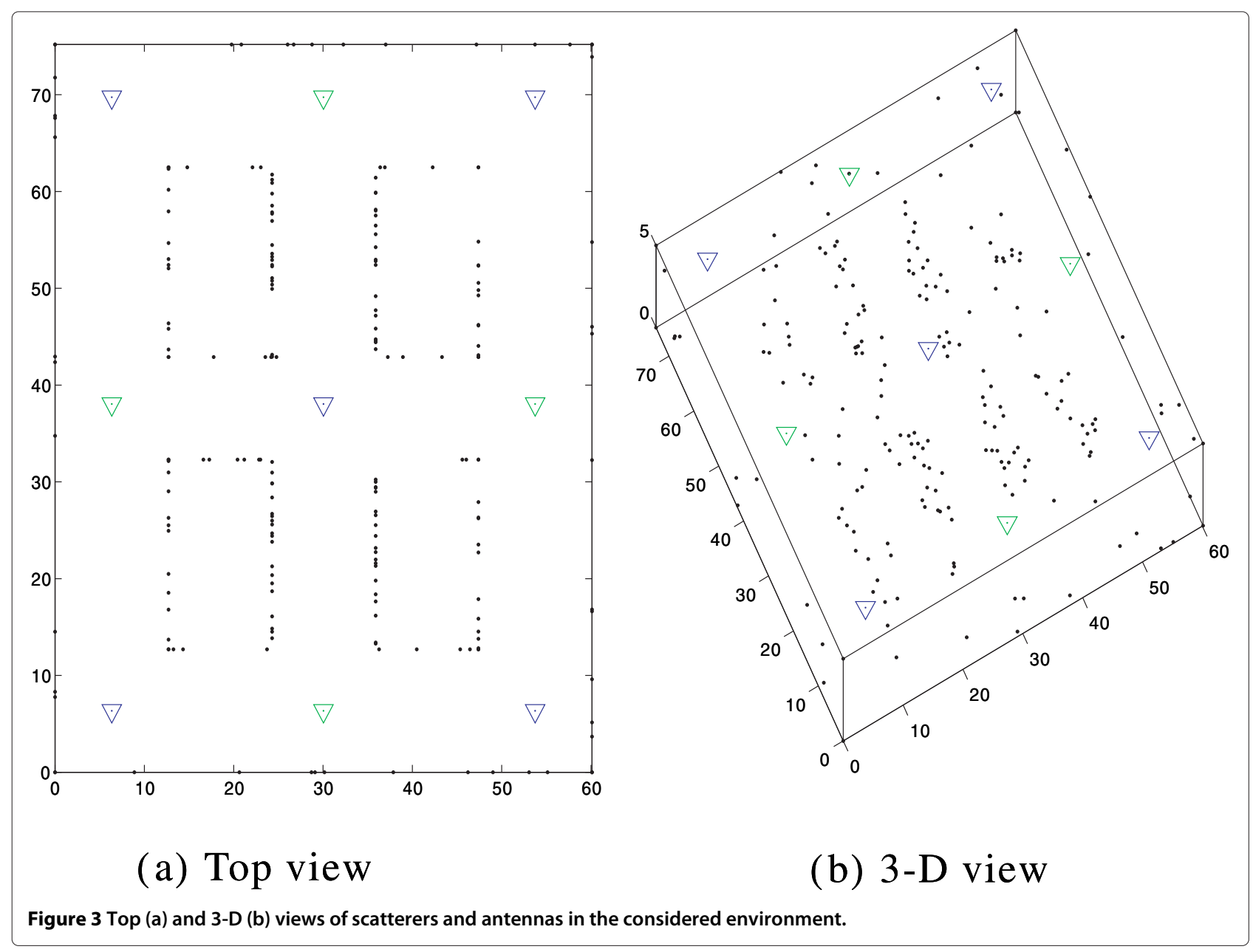


in Figure 3a. Scatterers are randomly distributed either on the inner walls of the hall or on the outer walls of the inside structures (See Figure 3b for the locations of the scatterers) to emulate the multi-reflection and diffuse scattering. There are also some scatterers located at the corners of the walls to emulate the diffraction. The parameters used in the graph modeling approach are listed in Table 1.

This indoor environment can be categorized as the optional A1 scenario specified in WINNER II SCM models [14]. To evaluate modeling accuracy using propagation graphs, two large-scale channel parameters, i.e., path loss and delay spread are chosen for comparison between the graph models and the standard WINNER II models. Totally, 12,384 IRs are generated using the propagation graphs for 1,376 UE locations, i.e., nine graphs per UE location. According to the visibility between the UE and the antennas, the IRs are split into the LoS group and the NLoS group. The path losses and delay spreads are computed for the two groups separately. Figure 4a,b depict the path loss versus distance in base10 logarithm of meters for LoS and NLoS, respectively. The regression lines fitted to the simulated samples are also illustrated. It can be observed that the slope of the line equals 16 for LoS and 20.96 for NLoS. According to the WINNER II models, this parameter equals 18.7 for LoS and 20 for NLoS (see p. 44 in [14]). Therefore, we consider that the results obtained with graph models are sufficiently close to the standardized model parameters.

Figure 5 depicts the cumulative distribution functions (CDFs) of the delay spreads calculated, respectively, from the individual snapshots of the LoS and NLoS scenarios generated by the graph models. It can be seen that the delay spreads in logarithm can be well characterized by Gaussian distributions, the means and variances are

Table 1 Parameter settings for graph-based IR simulations

\begin{tabular}{lc}
\hline Description & Value \\
\hline Carrier frequency & $2.6 \mathrm{GHz}$ \\
Bandwidth & $20 \mathrm{MHz}$ \\
Heights of Tx antennas & $4.6 \mathrm{~m}$ \\
Height of scatterers & {$[0,5] \mathrm{m}$} \\
Heights of UEs & $1.7 \mathrm{~m}$ \\
Total UE locations & 1,376 \\
Snapshots per location & 150 \\
Signal to noise ratio & $30 \mathrm{~dB}$ \\
Number of Tx antennas & 9 \\
Number of UE antennas & 1 \\
Groups of Tx antennas & 2 \\
Reflection gain & 0.8 \\
\hline
\end{tabular}

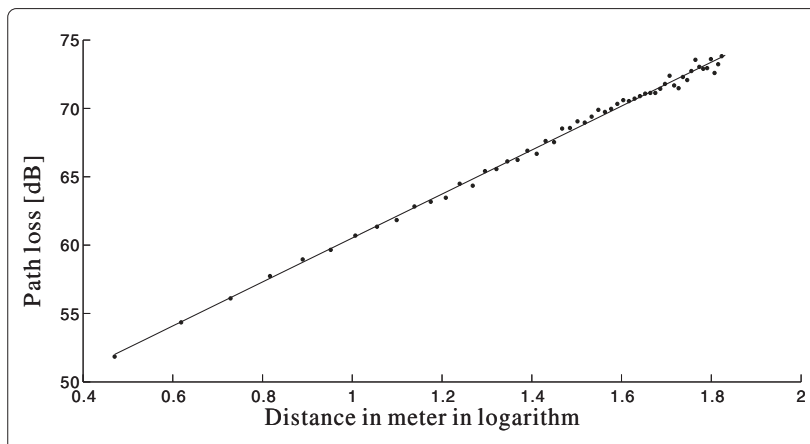

(a) $\operatorname{LoS}$

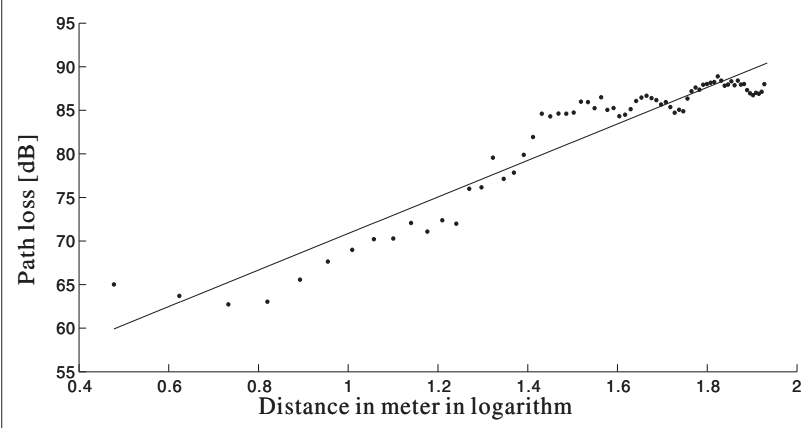

(b) NLoS

Figure 4 Path loss of channels simulated in indoor LoS (a) and NLoS (b) environment. Regression line $P=16.08 \log _{10} d+44.45$ is used to fit the results in LoS, and $P=20.96 \log _{10} d+49.90$ is used to fit the results in NLOS.

also quite close to the standard values presented in the WINNER II models (see p. 47 in [14]).

The above results demonstrate that for the characteristics of path loss and delay spread, graph modelingbased approaches can accurately reproduce the model parameters. Furthermore, in [13], the observations based on simulation show the feasibility of applying random graphs to propagation channel modeling in the spacetime-frequency domain. Moreover, the above consistencies are obtained based on the appropriate settings of the parameters in propagation graph modeling, such as reflection gain and distribution of the scatterers. Without loss of generality, the reflection gain is set to be 0.8 in indoor environment according to [8]. We also try other values in order to investigate how sensitive the delay spread is to the model parameter. Figure 6 shows the mean of delay spread versus the reflection gain. It can be clearly observed that in both LoS and NLoS scenarios, the mean of delay spread increases along with the reflection gain. That is reasonable as the higher the reflection gain, the slower the amplitude of IR decays, resulting in larger delay spread. These observations indicate that for different environments, realistic channel characteristics can be 


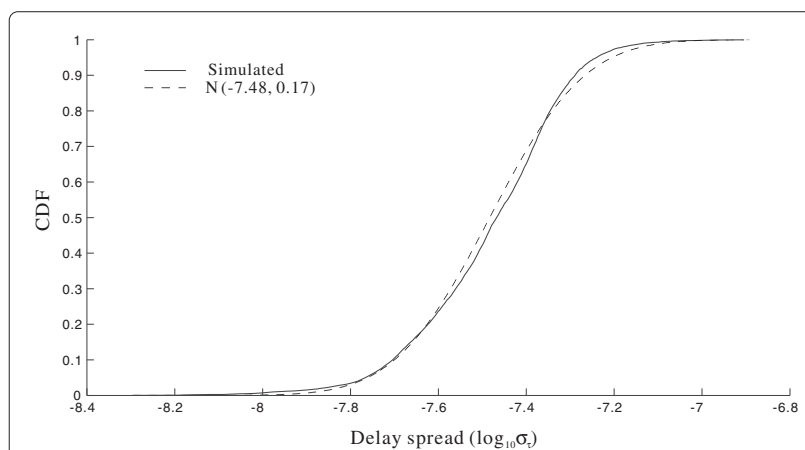

(a) LOS

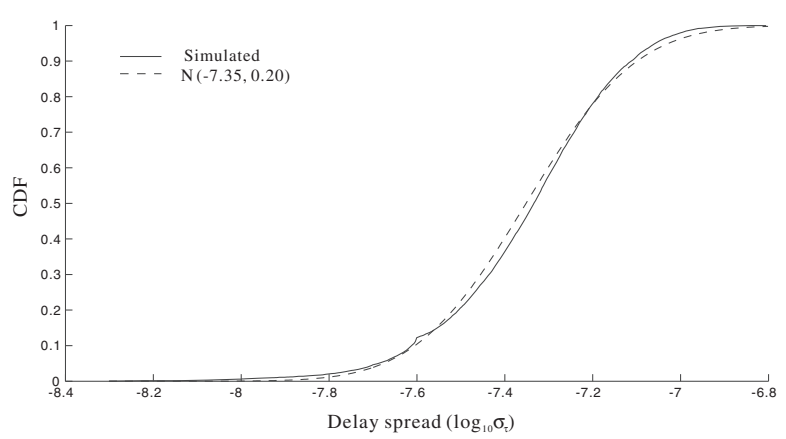

(b) NLOS

Figure 5 Delay spreads in logarithm for channels simulated in indoor LoS (a) and NLoS (b) environment respectively.

predicted by properly tuning the parameters of the graph models.

\section{Definition of spatial fading cross-correlation}

The spatial fading cross-correlation coefficient $\rho$ is defined as the cross-correlation coefficients of the

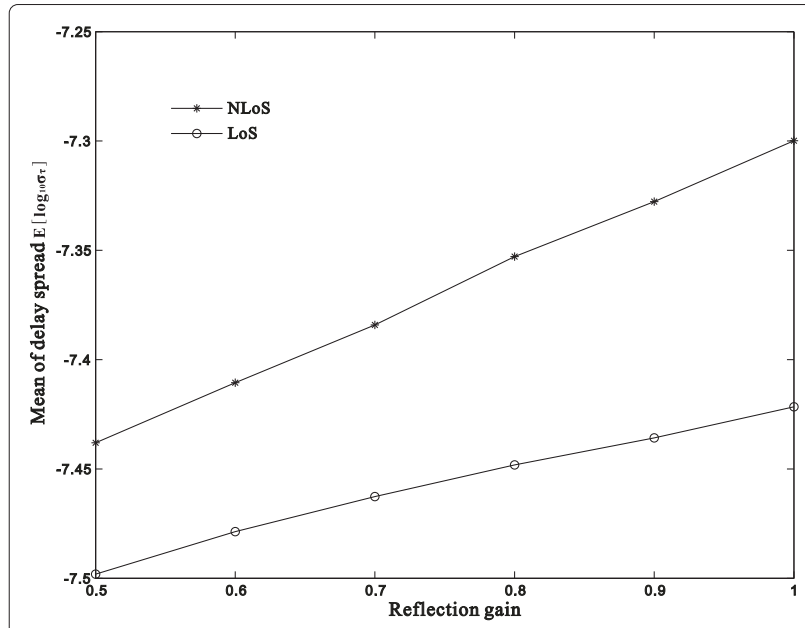

Figure 6 Mean of delay spread in logarithm versus reflection gain in indoor scenario. narrowband ${ }^{\text {a }}$ fading of links ' 1 ' and ' 2 ' observed at the same UE location, i.e.,

$$
\rho=\frac{E\left[\left(h_{1}-\overline{h_{1}}\right)\left(h_{2}-\overline{h_{2}}\right)^{*}\right]}{\sqrt{E\left[\left|h_{1}-\overline{h_{1}}\right|^{2}\right]} \sqrt{E\left[\left|h_{2}-\overline{h_{2}}\right|^{2}\right]}}
$$

where $h_{1}$ and $h_{2}$ denote the complex narrowband channel coefficients of two links, respectively, as shown in Figure 7 and $\bar{h}$ is the mean of the channel coefficient. $E\{\cdot\}$ denotes the expectation operation. Defining the mean-removed channel coefficients $\hat{h_{1}}=h_{1}-\overline{h_{1}}$ and $\hat{h_{2}}=h_{2}-\overline{h_{2}}$, the covariance $E\left[\left(h_{1}-\overline{h_{1}}\right)\left(h_{2}-\overline{h_{2}}\right)^{*}\right]$ in Equation 3 can be calculated as:

$$
\begin{aligned}
C_{1,2}= & E\left[\hat{h}_{1} \hat{h}_{2}^{*}\right] \\
= & E\left[\int_{T} \hat{h}_{1}(\tau) d \tau \int_{T} \hat{h}_{2}^{*}\left(\tau^{\prime}\right) d \tau^{\prime}\right] \\
= & E\left[\iint_{T} \hat{h}_{1}(\tau) \hat{h}_{2}^{*}(\tau) d \tau^{2}\right. \\
& \left.+\iint_{T, \tau \neq \tau^{\prime}} \hat{h}_{1}(\tau) \hat{h}_{2}^{*}\left(\tau^{\prime}\right) d \tau d \tau^{\prime}\right],
\end{aligned}
$$

where $h(\tau)$ denotes the complex-valued spread function of the channel in delay and $T$ is the observing time of each snapshot.

From Equation 4, it is clear that $C_{1,2}$ is split into two parts, i.e., the common-delay (cmd) part and cross-delay (crd) part. The narrowband fading cross-correlation coefficient $\rho$ can then be written as:

$$
\begin{aligned}
\rho & =\frac{C_{1,2}}{\sqrt{E\left[\left|\hat{h}_{1}\right|^{2}\right]} \sqrt{E\left[\left|\hat{h}_{2}\right|^{2}\right]}} \\
& =\frac{E\left[\iint_{T} \hat{h}_{1}(\tau) \hat{h}_{2}^{*}(\tau) d \tau^{2}\right]+E\left[\iint_{T, \tau \neq \tau^{\prime}} \hat{h}_{1}(\tau) \hat{h}_{2}^{*}\left(\tau^{\prime}\right) d \tau d \tau^{\prime}\right]}{\sqrt{E\left[\left|\hat{h}_{1}\right|^{2}\right.} \sqrt{E\left[\left|\hat{h}_{2}\right|^{2}\right]}} \\
& =\rho_{\text {cmd }}+\rho_{\text {crd }},
\end{aligned}
$$

where the discrete equivalents of cross-correlation coefficients of the cmd components $\rho_{\text {cmd }}$ and crd components $\rho_{\text {crd }}$ calculated in reality are expressed as:

$$
\rho_{\mathrm{cmd}}=\frac{\sum_{i=1}^{I} \sum_{n=1}^{N} \hat{h}_{1, i}\left(\tau_{n}\right) \hat{h}_{2, i}^{*}\left(\tau_{n}\right)}{\sqrt{\sum_{i=1}^{I} \sum_{n=1}^{N}\left|\hat{h}_{1, i}\left(\tau_{n}\right)\right|^{2}} \sqrt{\sum_{i=1}^{I} \sum_{n=1}^{N}\left|\hat{h}_{2, i}\left(\tau_{n}\right)\right|^{2}}}
$$

and

$$
\rho_{\mathrm{crd}}=\frac{\sum_{i=1}^{I} \sum_{n^{\prime}=1, n^{\prime} \neq n}^{N} \sum_{n=1}^{N} \hat{h}_{1, i}\left(\tau_{n}\right) \hat{h}_{2, i}^{*}\left(\tau_{n^{\prime}}\right)}{\sqrt{\sum_{i=1}^{I} \sum_{n=1}^{N}\left|\hat{h}_{1, i}\left(\tau_{n}\right)\right|^{2}} \sqrt{\sum_{i=1}^{I} \sum_{n=1}^{N}\left|\hat{h}_{2, i}\left(\tau_{n}\right)\right|^{2}}},
$$




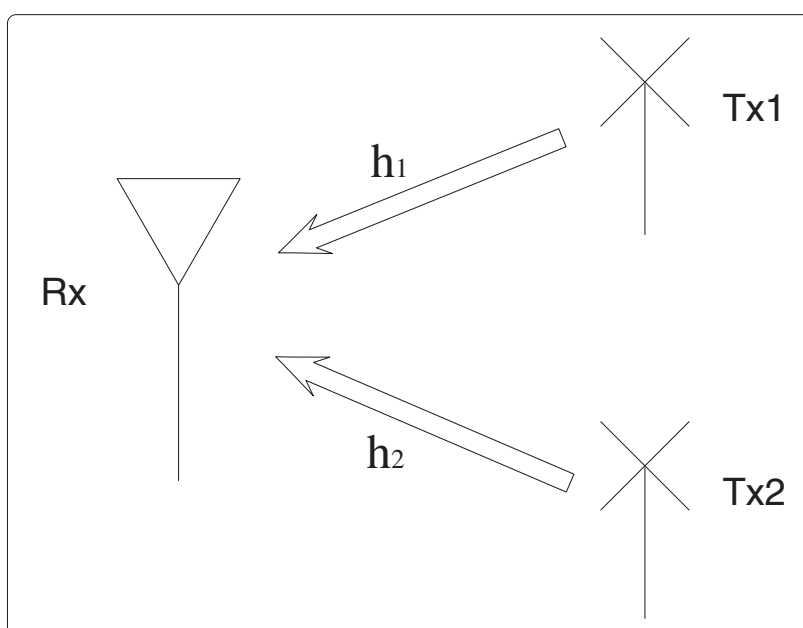

Figure 7 Propagation channels of a $2 \times 1$ DAS.

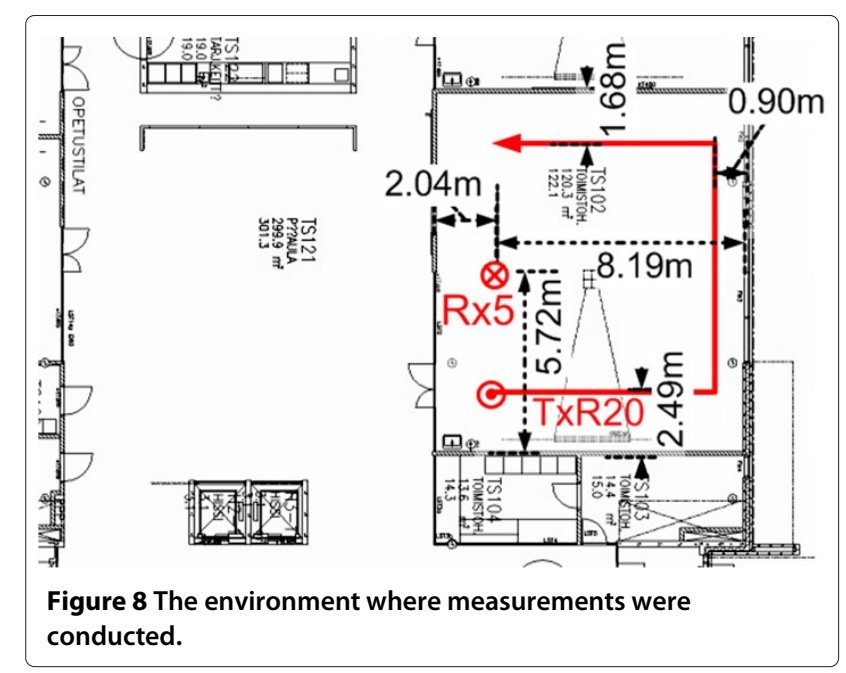

differences in small scale such as the movement of the person pushing the Tx trolley and the absorption give rise to the randomness in the observations of the channel. Since the transmitter and the receiver were equipped with 50 and 32 antennas, respectively, for each cycle in total, $50 \times 32=1,600$ channel coefficients are obtained. These channel observations are used to calculate the fading correlation coefficients. Figure 9 illustrates the contour plots of $\rho$ and $\rho_{\text {cmd }}$ computed based on the data of 150 cycles, while the contour plot and the CDF of the deviations between $\rho$ and $\rho_{\text {cmd }}$ are depicted in Figure 10. It can be observed that most of the deviations between $\rho$ and $\rho_{\text {cmd }}$ are close to zero. More than $97 \%$ of the deviations are less than 0.1 , and the mean value of $\rho-\rho_{\text {cmd }}$ is about 0.02 . Thus, $\left|\rho \approx \rho_{\text {cmd }}\right|$ can be considered empirically valid in indoor environments.

\section{Modeling spatial cross-correlation of fading in a DAS}

We are interested at the spatial cross-correlation of the fadings of two composite channels when nine antennas are grouped into two clusters. Figure 2 depicts an indoor environment where a DAS with nine antennas is deployed. The corresponding locations of transmitters and scatterers are depicted in Figure 3. The antennas are allocated in different parts of the room in nine positions. These antennas can be flexibly combined into two groups. An example illustrated in Figure 3 shows two groups of antennas marked in different colors. The graph modeling method is used to simulate the IRs of the composite channels when the UE is located at each position in the environment. The parameter settings for the simulations are shown in Table 1. In order to have enough number of random realizations of the fading coefficients, for an individual UE position, 150 channel IRs are generated with the UE moving randomly in 150 locations in the vicinity of 

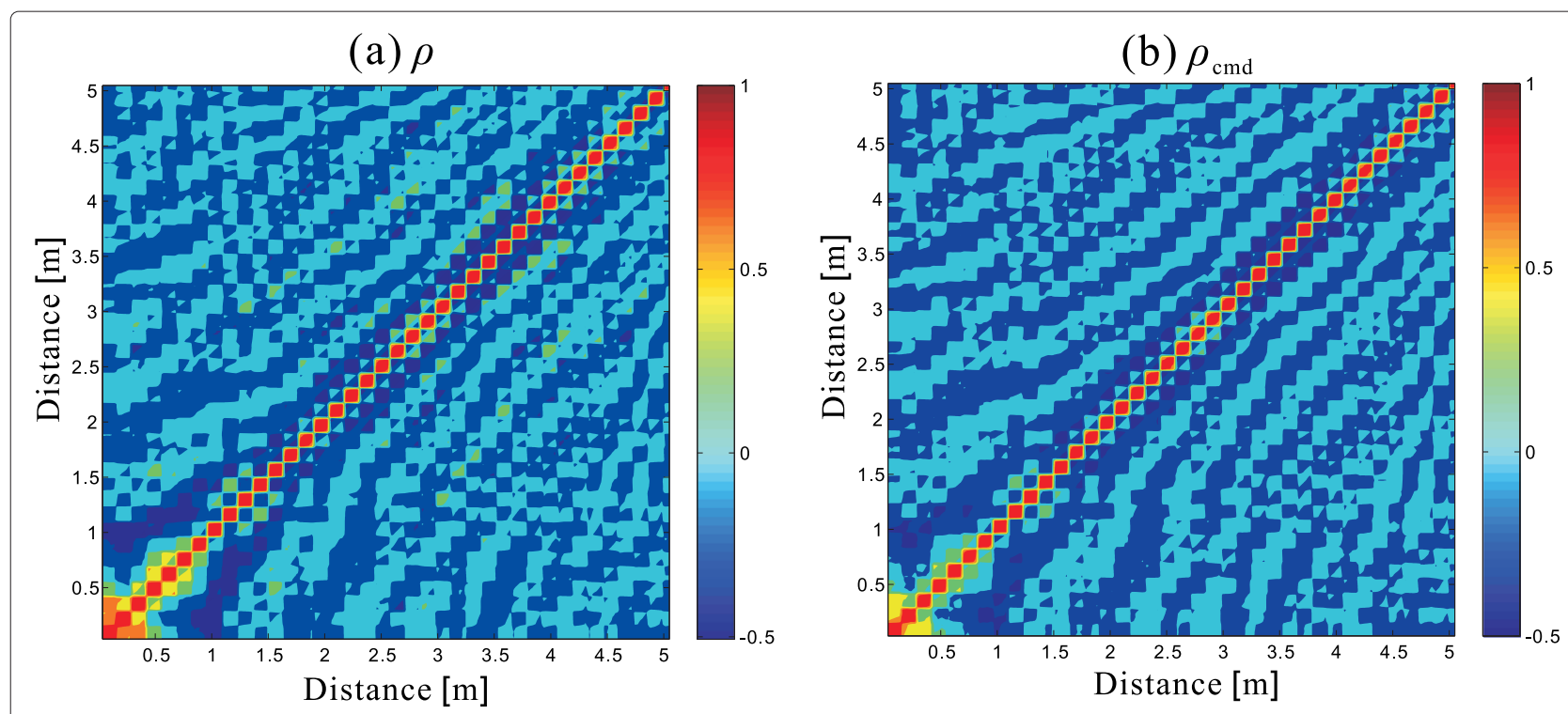

Figure 9 Contour plot of the experimental cross-correlation coefficients. (a) depicts the coefficients of narrowband channels, and (b) depicts the coefficients of wideband common-delay components in IRs.

this position within $\lambda / 4$. This vicinity range is determined accordingly such that random realizations are in the same WSS condition. Then the fading correlation is calculated based on the simulated IRs.

Figure 11 depicts the absolute values of fading crosscorrelation observed at the UE when the composite channels are generated using four different grouping schemes. In each scheme, groups $\mathrm{A}$ and $\mathrm{B}$ of antennas are specified. We use 'A:1 $-2-3$ ' to denote that the group A consists of the antennas 1,2 , and 3 . It can be observed that the fading cross-correlation exhibits symmetric pattern in accordance with the antenna constellations as well as the geometry of the environment. Significant spatial cross-correlation can be observed at locations where groups A and B antennas are distributed symmetrically. This is reasonable as the propagation channels observed by the UE from two nearest antennas, which are both in the LoS positions to the UE, are very similar, e.g., as in the case where the UE is in the middle of two antennas which belong to different groups. Low spatial fading

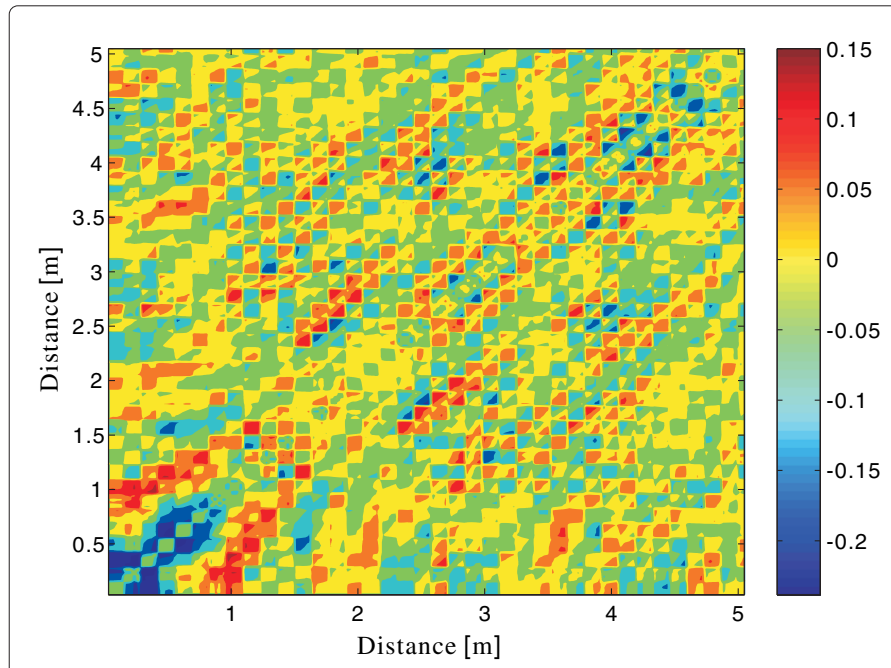

(a) $\rho-\rho_{\text {cmd }}$

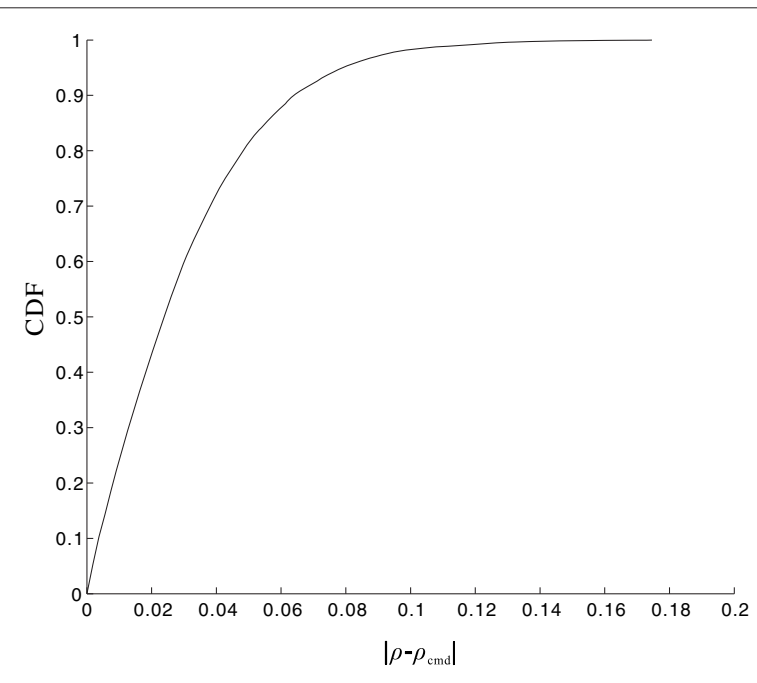

(b) CDF of $\left|\rho-\rho_{\text {cmd }}\right|$

Figure 10 Contour plot (a) and CDF (b) of deviation. Deviation between cross-correlation coefficients of narrowband channels and wideband common-delay components in IRs. 


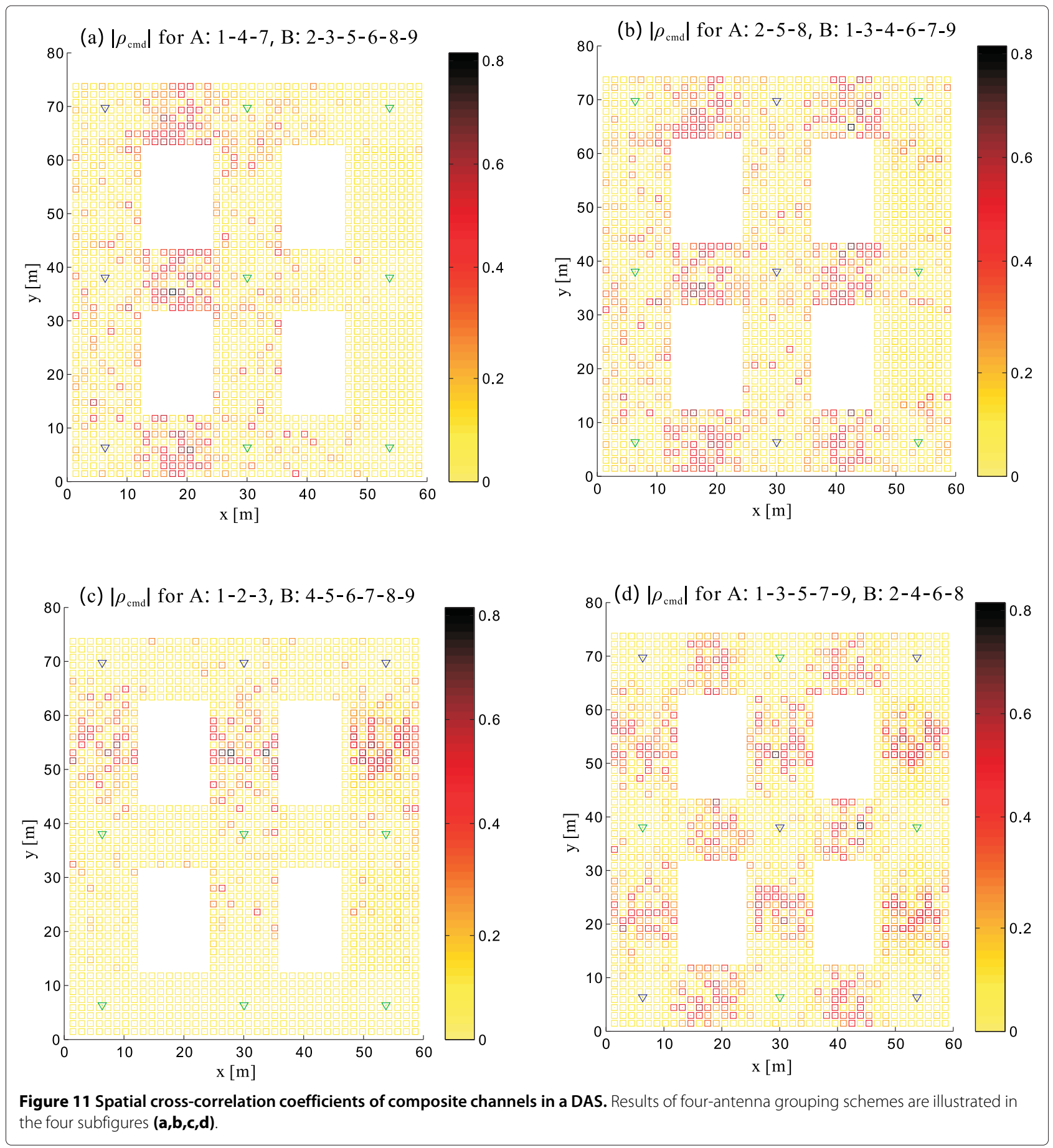

cross-correlation is observed at the locations where the groups $\mathrm{A}$ and $\mathrm{B}$ antennas are asymmetric with respect to (w.r.t.) the UE, e.g., when UE is located in the vicinity of the antennas. These observations hold for all four constellations illustrated in Figure 11. The CDFs of the fading cross-correlation coefficients in the four schemes are depicted in Figure 12. It can be observed that the configuration with locations of antennas in different groups overlapped gives the highest cross-correlation coefficients. When antennas belonging to different groups are separated, the cross-correlation becomes less.

From these results, it is apparent that the locations of antennas in the DAS lead to different fading crosscorrelation distribution in the area covered by the DAS. Thus, it is necessary to design the DAS and its transmission algorithms by considering the empirical 


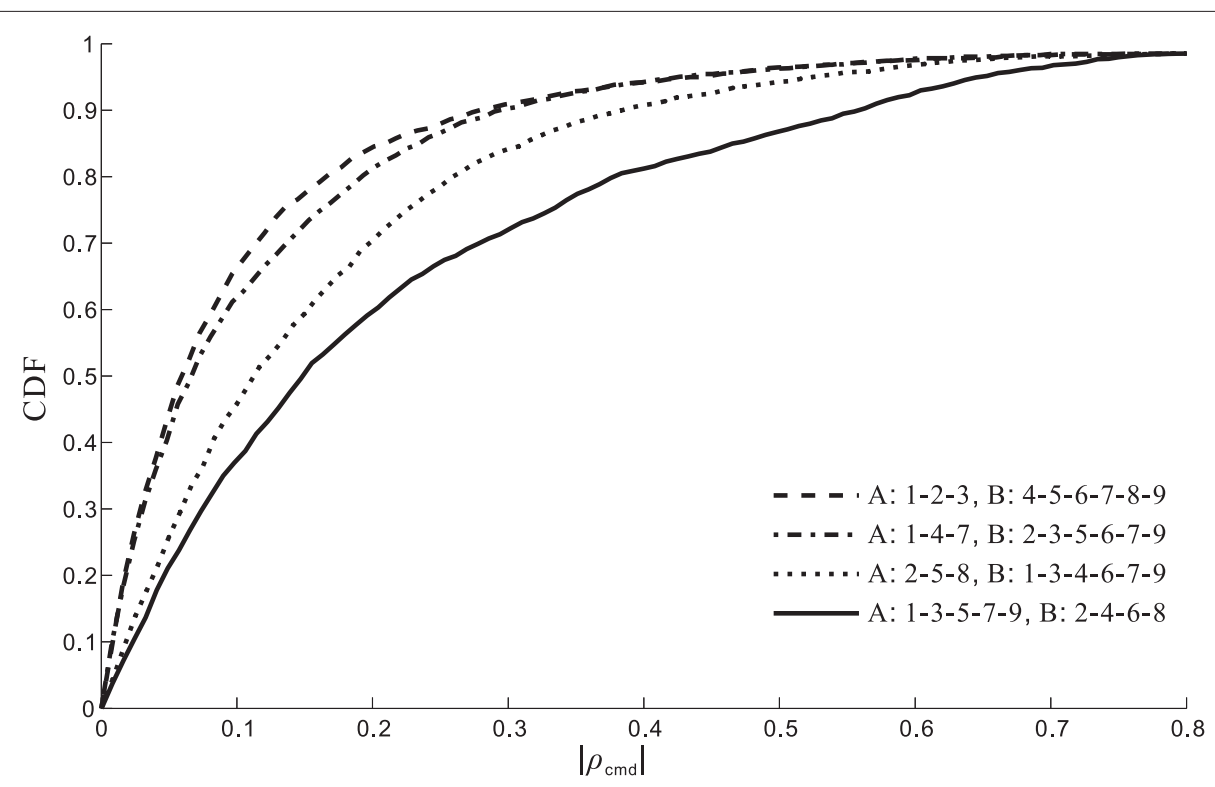

Figure 12 Cumulative distribution function of spatial cross-correlation coefficients of different groups in simulated DAS.

fading correlation in the environments of interest. A common belief for achieving better coverage and lowpower consumption is to allocate antenna symmetrically for the DAS $[1,2,16] \mathrm{y}$. However, the investigation results illustrated here show that the improvement by utilizing the DAS is marginal due to the non-negligible cross-correlation attributed to the symmetric antenna constellations.

\section{Experimental evaluation}

The spatial correlation in most of the DASs can be ignored because the spacing of the antennas are much larger than the coherent distance. However, in section 4, the simulation results show that significant fading cross-correlation can be observed in the case where two transmitting antennas are distributed in symmetric locations w.r.t. the UE. In this section, we use measurement data collected in the same campaign described in section 3.1 to validate this observation.

Figure 13 depicts a measurement environment where the locations of two distributed Txs are confined, respectively, in the two marked ranges that are symmetrical w.r.t. the Rx's location along the dashed line. Furthermore, the environments surrounding the Txs are also symmetric w.r.t. the dashed line. In order to evaluate the conclusions drawn from the simulation results in section 4, multiple Tx pairs with image locations to the $\mathrm{Rx}$ are considered to construct $2 \times 1$ DASs. Knowing the exact cycle indices at the corner points, the IRs of 50 cycles collected in the marked regions are identified for these $2 \times 1$ DASs. Figure 14a illustrates the correlations of these selected cycles. It can be observed that both the measured and simulated correlation coefficients $\rho$ are around 0.3 which are non-negligible in computing the diversity gain of the DAS.

In addition, the empirical statistics of the DAS channel correlation are extracted based on the measurements. Figure 14b depicts the empirical and simulated CDFs of the absolute values of fading correlation coefficients for symmetric links only and all links. It can be observed from Figure $14 \mathrm{~b}$ that $90 \%$ of the correlation coefficients for the measured symmetric links are larger than 0.3 , while the

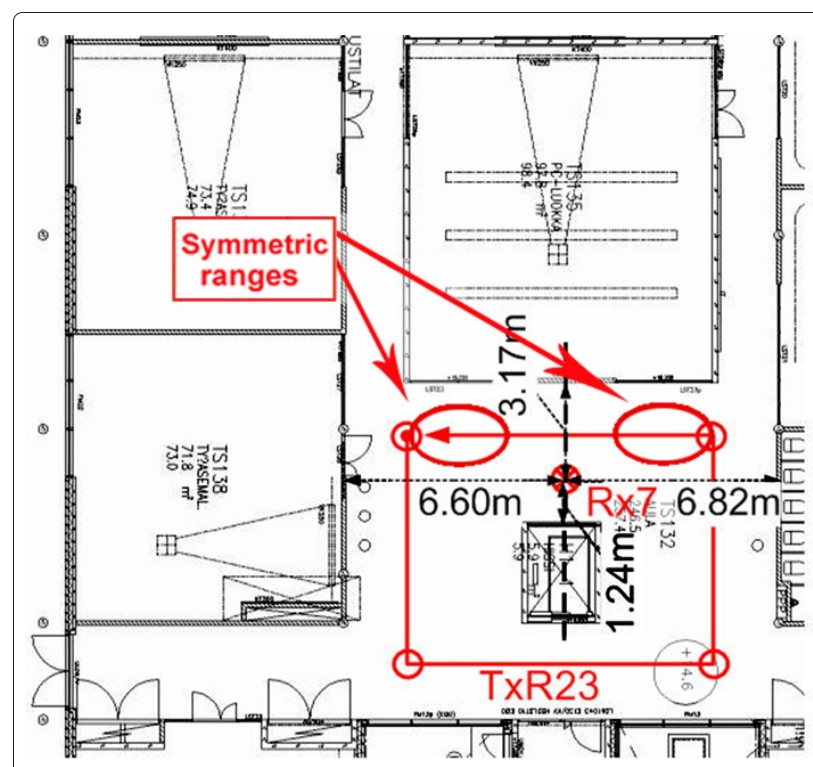

Figure 13 A symmetric environment where measurement was conducted. 

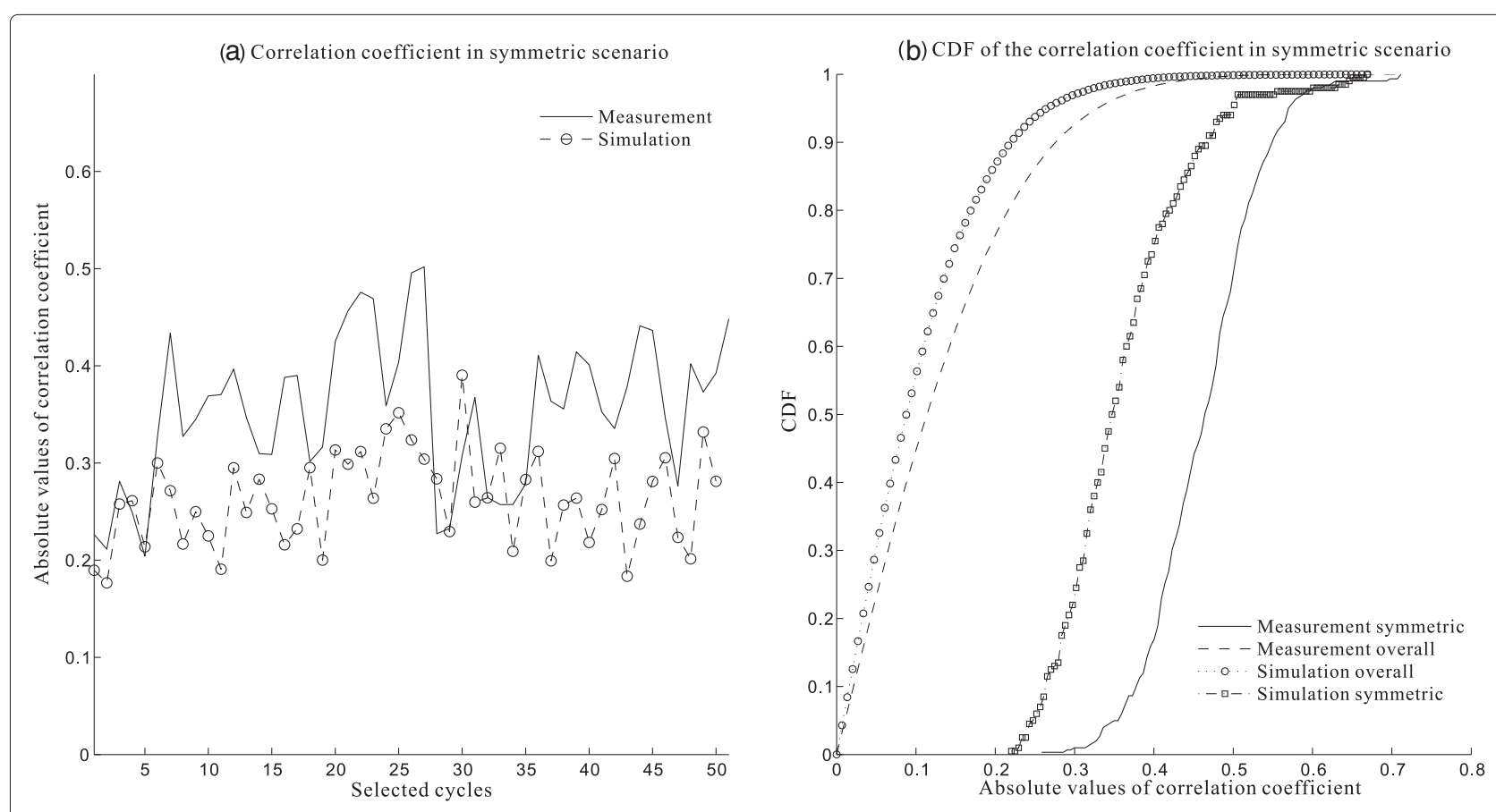

Figure 14 Fading correlation coefficients (a) and their CDFs (b) in specified DAS constellations. The solid line depicts the fading correlation of the links which poses the symmetric pattern, and the dash line represents the fading correlation of all the links based on measurement. Similarly, the symmetric and overall results based on simulation are depicted in squares and circles, respectively.

correlation coefficients of all links are much less and negligible. Similar results are observed for the simulations, even though the correlation coefficients are underestimated in the simulations. It is our conjecture that the underestimation is due to the settings of random phase in Equation 2 for graph modeling approach which might not hold in realistic environment.

\section{Conclusions}

The spatial cross-correlation of channel fadings in different links of a DAS has been investigated via simulations using stochastic propagation graphs. We first proposed an approach for computing the fading crosscorrelation, i.e., it is approximated by integrating the cross-correlation coefficients of the small-scale fadings in the same delay bins of two channels. The effectiveness of this approach has been evaluated using the measurement data. Then, the applicability of random propagation graphs in channel modeling was validated. It has been shown that the statistics of path loss and delay spreads extracted using graphs is close to those specified by the WINNER II models. Using the proposed methods, the narrowband fading cross-correlation of the composite channels in DASs for a hall-alike indoor environment has been investigated. The results obtained demonstrated that the highest cross-correlation coefficients can be observed when the locations of antennas in different groups are overlapped. When antennas belonging to different groups are located in two well-separated regions, the crosscorrelation becomes smaller. Moreover, significant fading cross-correlation can be observed in the cases where the distributed antennas belonging to different groups are deployed symmetrically w.r.t. the location of the UE. These results have been shown to be consistent with the observations obtained in real measurements.

\section{Endnote}

${ }^{a}$ Here, narrowband means that signal bandwidth times delay spread is much smaller than 1 .

\section{Competing interests}

The authors declare that they have no competing interests.

\section{Acknowledgments}

This work is supported by the fundamental project of the Science and Technology Commission of Shanghai Municipality (10ZR1432700, Multidimensional power spectrum characterization and modeling for wide-band propagation channels), the China Education Ministry "New-teacher" Project (20090072120015, Time-Variant Channel Characterization, Parameter Estimation and Modeling), Central Higher Education Fundamental Research Project (Polarization characteristics of propagation channel), the fundamental project of the Science and Technology Commission of Shanghai Municipality (13510711000, System design and demo-construction for cooperative networks of high-efficiency $4 \mathrm{G}$ wireless communications in urban hot-spot environments), and Central Higher Education Fundamental Research Project (ROF based distributed MIMO systems). The authors wish to acknowledge Dr. Junhe Zhou from Tongji University for his enlightening advices and valuable comments on the paper, and Elektrobit, Finland for kindly providing the measurement data. Part of the works in this paper has been presented in the COST IC1004 + iPLAN Joint Workshop on "Small Cell Cooperative Communications". 


\section{References}

1. W Roh, High performance distributed antenna cellular networks. PhD thesis, Dept. Elect. Eng., Stanford Univ., Stanford, CA 2003.

2. HXia, A Herrera, S Kim, F Rico, A CDMA-distributed antenna system for in-building personal communications services. IEEE J. Selected Areas Commun. 14(4), 644-650 (1996). IEEE, Piscataway

3. T Alade, $\mathrm{H}$ Zhu, H Osman, in IEEE 22nd International Symposium on Personal Indoor and Mobile Radio Communications (PIMRC) Spectral efficiency analysis of distributed antenna system for in-building wireless communication (IEEE, Piscataway, 2011), pp. 2075-2079

4. J Lee, J Roh, J Kwun, C Kang, in IEEE International Conference on Universal Personal Communications (ICUPC), vol. 1 A controlled distributed antenna system for increasing capacity in the DS-CDMA system (IEEE, Piscataway, 1998), pp. 345-348

5. T Ahmad, S Al-Ahmadi, H Yanikomeroglu, G Boudreau, in IEEE 73rd Vehicular Technology Conference (VTC Spring) Downlink linear transmission schemes in a single-cell distributed antenna system with port selection (IEEE, Piscataway, 2011), pp. 1-5

6. W Roh, A Paulraj, in IEEE 56th Vehicular Technology Conference (VTC Fall), vol.3 Outage performance of the distributed antenna systems in a composite fading channel (IEEE, Piscataway, 2002), pp. 1520-1524

7. X Zhou, X Yin, BJ Kwak, HK Chung, in Proceedings of 5 th European Conference on Antennas and Propagation (EuCAP) Experimental investigation of impact of antenna locations on the capacity of wideband distributed antenna systems in indoor environments (IEEE Piscataway, 2011), pp. 1639-1643

8. T Pedersen, B Fleury, in IEEE International Conference on Communications (ICC) Radio channel modelling using stochastic propagation graphs (IEEE Piscataway, 2007), pp. 2733-2738

9. T Fugen, J Maurer, T Kayser, W Wiesbeck, Capability of 3-D ray tracing for defining parameter sets for the specification of future mobile communications systems. IEEE Trans. Antennas Propagation. 54(11), 3125-3137 (2006). IEEE, Piscataway

10. Y Lostanlen, G Gougeon, in International Conference on Electromagnetics in Advanced Applications (ICEAA) Introduction of diffuse scattering to enhance ray-tracing methods for the analysis of deterministic indoor UWB radio channels (Invited Paper) (IEEE Piscataway, 2007), pp. 903-906

11. V Degli-Esposti, D Guiducci, A de'Marsi, P Azzi, F Fuschini, An advanced field prediction model including diffuse scattering. IEEE Transact. Antennas Propagation. 52(7), 1717-1728 (2004). IEEE, Piscataway

12. T Pedersen, G Steinbock, BH Fleury, Modeling of reverberant radio channels using propagation graphs. IEEE Trans. Antennas Propagation. 60(12), 5978-5988 (2012). IEEE, Piscataway

13. L Tian, X Yin, Q Zuo, J Zhou, Z Zhong, SX Lu, in IEEE 23rd International Symposium on Personal Indoor and Mobile Radio Communications (PIMRC) Channel modeling based on random propagation graphs for high speed railway scenarios (IEEE Piscataway, 2012), pp. 1746-1750

14. P Kyosti, J Meinila, L Hentila, X Zhao, T Jamsa, C Schneider, M Narandzic, M Milojevic, A Hong, Ylitalo J, V-M Holappa, M Alatossava, R Bultitude, Y de Jong, T Rautiainen, Winner II channel models (d1.1.2v1.1). (2007). http:// www.ist-winner.org/WINNER2-Deliverables/D1.1.2v1.1.pdf. Accessed Nov 2007

15. N Czink, The random-cluster model - a stochastic MIMO channel model for broadband wireless communication systems of the 3rd generation and beyond. PhD thesis, Technische Universitat Wien, Vienna, Austria, FTW Dissertation Series, 2007.

16. X Hu, Y Zhang, Y Jia, S Zhou, L Xiao, in Fourth International Conference on Communications and Networking in China (ChinaCOM) Power coverage and fading characteristics of indoor distributed antenna systems (IEEE Piscataway, 2009), pp. 1-4

doi:10.1186/1687-1499-2013-183

Cite this article as: Tian et al:: Spatial cross-correlation modeling for propagation channels in indoor distributed antenna systems. EURASIP Journal on Wireless Communications and Networking 2013 2013:183.

\section{Submit your manuscript to a SpringerOpen ${ }^{\mathcal{O}}$ journal and benefit from:}

- Convenient online submission

Rigorous peer review

- Immediate publication on acceptance

- Open access: articles freely available online

- High visibility within the field

- Retaining the copyright to your article

Submit your next manuscript at springeropen.com 